\title{
hsa-miR-24 suppresses metastasis in nasopharyngeal carcinoma by regulating the c-Myc/epithelial-mesenchymal transition axis
}

\author{
BOJIN SU ${ }^{1,2^{*}}$, TAO XU ${ }^{1-3^{*}}$, JEFF P. BRUCE ${ }^{4}$, KENNETH W. YIP ${ }^{4}$, NING ZHANG $^{3}$, ZELI HUANG $^{3}$, \\ GUOYI ZHANG ${ }^{3}$, FEI-FEI LIU ${ }^{4,5}$, JIYONG LIANG ${ }^{2}$, HUILING YANG ${ }^{1}$ and FRANÇOIS X. CLARET ${ }^{2,6}$
}

\author{
${ }^{1}$ Department of Pathophysiology, Zhongshan School of Medicine, Sun Yat-sen University, Guangzhou, \\ Guangdong 510080, P.R. China; ${ }^{2}$ Department of Systems Biology, The University of Texas MD Anderson Cancer Center, \\ Houston, TX 77030, USA; ${ }^{3}$ Department of Radiation Oncology, Cancer Center, First People's Hospital of Foshan, \\ Foshan, Guangdong 528000, P.R. China; ${ }^{4}$ Department of Medical Biophysics, University of Toronto, Toronto, \\ ON M5G 1L7; ${ }^{5}$ Department of Radiation Oncology, Princess Margaret Cancer Centre, University Health Network, \\ Toronto, ON M5G 2M9, Canada; ${ }^{6}$ Experimental Therapeutics Academic Program and Cancer Biology Program, \\ The University of Texas Graduate School of Biomedical Sciences at Houston, Houston, TX 77030, USA
}

Received March 27, 2018; Accepted July 19, 2018

DOI: $10.3892 /$ or.2018.6690

\begin{abstract}
Distant metastasis is the major contributor to treatment failure and mortality in patients with nasopharyngeal carcinoma (NPC). The lack of effective treatment strategies for metastatic NPC is the major cause for the low survival rate. Therefore, it is crucial to understand the molecular mechanisms underlying NPC metastasis and to identify potential biomarkers for targeted therapy. MicroRNA (miRNAs or miRs) have been shown to play an important role in tumorigenesis and metastasis. In the present study, we aimed to evaluate the significance of hsa-miR-24 in NPC metastasis. Significantly lower hsa-miR-24 levels were observed in NPC metastatic tumors and higher hsa-miR-24 levels were associated with longer progression-free
\end{abstract}

Correspondence to: Professor Huiling Yang, Department of Pathophysiology, Zhongshan School of Medicine, Sun Yat-sen University, 74 2nd Zhongshan Road, Guangzhou, Guangdong 510080, P.R. China

E-mail: yanghl@mail.sysu.edu.cn

Professor Francois X. Claret, Department of Systems Biology, The University of Texas MD Anderson Cancer Center, 7435 Fannin Street, Unit 0950, Houston, TX 77030, USA

E-mail: fxclaret@mdanderson.org

*Contributed equally

Abbreviations: NPC, nasopharyngeal carcinoma; miRNAs or miRs, microRNAs; EMT, epithelial-mesenchymal transition; FFPE, formalin-fixed paraffin-embedded; TNM, tumor site, lymph node, metastasis; TCGA, The Cancer Genome Atlas; HNSCC, head and neck squamous cell cancer; RT-qPCR, reverse transcription-polymerase chain reaction

Key words: nasopharyngeal carcinoma, microRNA, c-Myc, epithelial-mesenchymal transition, metastasis and metastasis-free survival durations. hsa-miR-24 overexpression inhibited cell proliferation, invasion and migration. Using bioinformatics approaches together with functional luciferase reporter assays, we demonstrated that the c-Myc 3'-UTR was a direct target of hsa-miR-24 in regulating NPC metastasis. Protein profiling analysis revealed that a high c-Myc expression was inversely associated with metastasis-free overall survival and with epithelial-mesenchymal transition (EMT). Furthermore, the overexpression of hsa-miR-24 decreased NPC cell invasive ability induced by the overexpression of c-Myc, associated with EMT epithelial marker (E-cadherin) restoration. Thus, on the whole, the findings of this study demonstrate that hsa-miR-24 suppresses metastasis in NPC by regulating the c-Myc/EMT axis, suggesting that hsa-miR-24 may be used as a prognostic factor and as a novel target for the prevention of NPC metastasis.

\section{Introduction}

Nasopharyngeal carcinoma (NPC) is an epithelial head and neck cancer; it is endemic mostly in Southeast Asia, the Middle East, North Africa, Alaska, Greenland, and the Mediterranean, with the highest incidence rate of NPC is amongst Cantonese living in Southern China (1,2). Radiation therapy is the main treatment for NPC and results in a favorable outcome in early-stage disease $(3,4)$. However, the 10 -year overall survival rate of patients with advanced-stage disease is $<50 \%$, and almost half of the patients experience treatment failure. More than $30 \%$ of stage III-IVA-B cases experience distant metastasis after combination therapy, which is the major contributor to mortality $(5,6)$. The lack of effective treatment strategies for metastatic NPC has led to the low survival rate. Thus, it is imperative to elucidate the molecular mechanisms that underlie NPC metastasis and identify potential therapeutic targets for NPC which may lead to novel treatment strategies.

MicroRNAs (miRNAs or miRs) are short non-coding RNAs (20-24 nucleotides in length) that regulate tumor 
development and cell differentiation, apoptosis and proliferation $(7,8)$. Moreover, miRNAs have emerged as key regulators of gene expression in almost all biological processes, including tumor metastasis. miRNAs have regulatory functions and may act as oncomiRs or tumor suppressor miRs in cancer initiation and progression (9-11). Accumulating evidence has indicated that the deregulation of miRNAs can affect cancer metastasis by regulating the metastatis-related genes and signaling pathways. In particular, miR-203 downregulation has been shown to promote metastasis, increasing target Snail2 expression, thereby increasing breast cancer cell invasion and migration (12); miR-9 upregulation has also been shown to induce a loss in E-cadherin expression and $\beta$-catenin signaling pathway activation, resulting in a high vascular endothelial growth factor (VEGF) expression and, consequently, in the promotion of tumor metastasis and angiogenesis (13). Of note, emerging evidence has indicated that manipulating miRNA expression is an effective strategy with which to suppress cancer metastasis; for example, the use of miR-21 and miR-10b antagomiRs has been shown to markedly suppress the formation of lung metastases by inhibiting miR-21 and miR-10b expression in colorectal cancer and breast cancer xenograft models, respectively $(14,15)$, which indicates that miRNA-based therapy is an attractive novel treatment strategy for cancer metastasis. However, whether specific miRNAs regulate tumor progression and metastasis and can therefore be exploited as biomarker requires further efforts to systematically evaluate the contribution of miRNAs to the metastatic process and identify their molecular targets with the underlying mechanisms.

Our previous studies indicated that hsa-miR-24 functions as a tumor suppressor as well as radiosensitizer in NPC (16-18); however its role in NPC metastasis remains unknown. Bruce et al (19) performed global miRNA expression profiling of two independent NPC cohorts and identified an miRNA signature that could predict distant metastasis in this disease; hsa-miR-24 was included in this signature; however, its role in the metastatic processes was not identified. This evidence suggests that the miR-24 signature is a promising target in NPC distant metastasis. However, the exact mechanisms through which hsa-miR-24 suppresses metastasis in NPC remain largely unknown. Thus, the understanding of the mechanisms through which hsa-miR-24 regulates metastasis may provide a target for the diagnosis and prognosis of patients with high-TNM grade NPC and may offer provide novel therapeutic approaches.

In the present study, we aimed to investigate the regulatory mechanisms of hsa-miR-24 in NPC metastasis. We demonstrated that hsa-miR-24 was a negative NPC metastatic factor. Furthermore, to the best of our knowledge, we demonstrate for the first time that that c-Myc is a direct target of hsa-miR-24, and that the overexpression of hsa-miR-24 in NPC cells decreases both the mRNA and protein expression of c- Myc. In addition, hsa-miR-24 suppressed the NPC cell invasive ability induced by c-Myc overexpression, and was associated with the restoration of the expression of E-cadherin, an epithelial marker. Thus, by regulating the c-Myc/EMT axis, hsa-miR-24 suppresses metastasis in NPC. Our finding revealed that hsa-miR-24 could be used as a prognostic factor and as a promising target for NPC treatment.

\section{Materials and methods}

Cell culture. The human NPC cell lines, 5-8F, 6-10B, and C666-1, were obtained from the Cancer Center of Sun Yat-sen University (Guangzhou, China). All cells were cultured in RPMI-1640 medium (Gibco, Grand Island, NY, USA) containing $10 \%$ fetal bovine serum, $0.5 \%$ penicillin-streptomycin sulfate, and incubated in a $37^{\circ} \mathrm{C}$ humidified incubator with $5 \% \mathrm{CO}_{2}$.

Ethics statement. All human tissues in this study were collected using protocols approved by the Ethics Committee of the First People's Hospital of Foshan (Foshan, China). The clinical and pathological data of the patients were analyzed anonymously. All tissue specimens were handled and made anonymous based on ethical and legal standards. All patients provided written informed consent prior to participation in this study.

Clinical data and tissue samples. This study included two independent patient-related databases for the study of hsa-miR-24, the training group and the validation group. The training group included 11 metastatic cases and 12 non-metastatic cases of formalin-fixed paraffin-embedded (FFPE) patient specimens which were obtained from the First People's Hospital of Foshan between January, 2011 and December, 2015. The validation group was an miRNA profiling analysis including 60 pairs of metastatic and non-metastatic NPC cases from the Princess Margaret Cancer Centre (Toronto, ON, Canada), and hsa-miR-24 expression was measured with nCounter Human miR Assay from NanoString Technologies (version 1.0), as previously described (19). In addition, reverse phase protein assay (RPPA) protein profiling of c-Myc expression was obtained from a previously described cohort (20). The clinical characteristics of all the patients, including tumor site, lymph node metastasis and tumor node metastasis (TNM) stage are summarized in Table I. The TNM stage was classified based on the 7th edition staging system of American Joint Committee on Cancer. Patients with metastatic disease at the time of diagnosis were excluded.

Cell transient transfection. The NPC cells were plated onto 6 -well plates using medium without antibiotics $24 \mathrm{~h}$ prior to transfection. mirVana hsa-miR-24 mimics (10, 30 and $60 \mathrm{nM}$, Ambion; Thermo Fisher Scientific, Inc., Waltham, MA, USA) or Pcbs-human-c-Myc plasmid (0.5 and $1.5 \mu \mathrm{g}$, Addgene, Cambridge, MA, USA) were then transfected into the cells using Lipofectamine 2000 (Invitrogen, Carlsbad, CA, USA) according to the manufacturer's instructions. Opti-MEM medium (Gibco) was replaced with regular culture medium without antibiotics following transfection for $6 \mathrm{~h}$. Following $48 \mathrm{~h}$ of transfection, the cells were harvested to extract protein and RNA. mirVana hsa-miR-24 mimics and mirVana mimic negative control were obtained from Ambion; Thermo Fisher Scientific, Inc.

Establishment of a hsa-miR-24-overexpressing cell line. An hsa-miR-24-overexpressing cell line was established using the Lentiviral miRNA Expression system was from Biosettia (San Diego, CA, USA) as previously described (17). Briefly, the C666-1 cells were seeded into a 12 -well plate $24 \mathrm{~h}$ prior 
Table I. Clinical characteristics of the patients with NPC from two independent databases.

$\begin{array}{cc}\begin{array}{c}\text { Training group } \\ \text { (Foshan data) }\end{array} & \text { Validation group } \\ \text { (PM center) }\end{array}$

\begin{tabular}{lrc}
\hline Sex & & \\
Male & 21 & 93 \\
Female & 2 & 27 \\
Age (years) & & \\
$\geq 50$ & 14 & 68 \\
$<50$ & 9 & 52 \\
T classification & & \\
T1-2 & 9 & 82 \\
T3-4 & 14 & 36 \\
Tx & 0 & 2 \\
N classification & & \\
N0-1 & 8 & 88 \\
N2-3 & 15 & 1 \\
Nx & 0 & \\
TNM staging & & 63 \\
I-II & 1 & 55 \\
III-IVA-B & 22 & 2 \\
NA & 0 & 60 \\
miR-24 expression & & 60 \\
Low & 11 & \\
High & 12 & \\
\hline
\end{tabular}

The training group included 11 metastatic cases and 12 non-metastatic cases of formalin-fixed paraffin-embedded patient specimens, obtained from the First People's Hospital of Foshan between January, 2011 and December, 2015. The validation group included miRNA profiling analysis from the Princess Margaret Cancer Centre, with 60 pairs of metastatic and non-metastatic nasopharyngeal carcinoma (NPC) cases.

to transfection with hsa-miR-LV-24-1 or miR-LV-control. The medium was replaced with new medium containing Polybrene, and miR-LV-24-1 or miR-LV-control was added followed by centrifugation at $1.000 \mathrm{x} \mathrm{g}$ for $40 \mathrm{~min}$ at room temperature. The cells were selected by puromycin at a concentration of $3 \mu \mathrm{g} / \mathrm{ml}$ (C666-1). The cells stably expressing hsa-miR-24 were named C666-1-miR-24. The hsa-miR-24 expression level was verified by RT-qPCR. An eRFP reporter is designed in the Lentiviral miRNA Expression system to monitor transduction efficiency under a microscope (RFP image), and a single colony was selected to determine the differential transduction efficiency of the cells stably overexpressing miR-24 or not.

Cell viability assay and colony formation assay. The cells stably expressing hsa-miR-24 were seeded into 96-well plates $(2,000$ cells/well). Cell viability was measured with PrestoBlue reagent (Invitrogen Life Technologies, Carlsbad, USA) at 2, 3, 4 and 5 days. The optical density at $570 \mathrm{~nm}$ was measured in a 96-well plate using a spectrophotometer (Thermo Fisher Scientific, Inc.), and data were analyzed using
GraphPad Prism software version 5.0 (GraphPad Software, La Jolla, CA, USA) to obtain the $\mathrm{IC}_{50}$ value. A colony formation assay was performed to analyze the growth of hsa-miR-24 stably expressing cells seeded in 6-well plates ( 400 cells/well). After 14 days, cell colonies were washed with PBS, and fixed for $15 \mathrm{~min}$ at room temperature. The cells were then stained with $0.1 \%$ crystal violet (Sigma-Aldrich Corp.; Merck KGaA, Darmstadt, Germany ) for $15 \mathrm{~min}$ at room temperature. Colony numbers were counted (colonies consisting of $\geq 50$ cells).

Wound-healing assay. The hsa-miR-24 stably expressing cells were seeded at a density of $1 \times 10^{5}$ cells/well in 6-well plates and incubated until $90 \%$ confluent. A scratch wound was made across the center of the monolayer of cells in each well using a sterile $200 \mu 1$ pipette tip. The cells were then incubated in medium without serum for $24 \mathrm{~h}$. Images of the cells that had migrated into the cell-free scratch wound area were acquired, and the migration distance was measured under phase-contrast optics (10X objective). The scratch wound widths were determined by the percentage of cells that had migrated into the initially cell-free scratch area compared with the untreated control cells.

Cell invasion assays. Tumor cell invasion assays were performed using a Transwell chamber (Corning Life Sciences, New York, NY, USA) with $8 \mu \mathrm{m}$ pores coated with Matrigel (BD Biosciences, Franklin Lakes, NJ, USA) in 24-well dishes. In brief, the hsa-miR-24 stably expressing cells (C666-1-miR-24; $1 \times 10^{5}$ ) were seeded in the upper chamber with $1 \%$ fetal bovine serum, and medium supplemented with $10 \%$ fetal bovine serum was placed in the lower chamber. The plates were incubated at room temperature for $24 \mathrm{~h}$, and the cells were then fixed with $10 \%$ methanol and $0.1 \%$ crystal violet for $15 \mathrm{~min}$ at ambient temperature. The cells on the upper side of the filters were removed using cotton-tipped swabs, and the filters were washed with PBS and observed under an inverted microscope (Axio Observer Z1, Carl Zeiss.Inc, Oberkochen, Germany).

Western blot analysis. A western blot analysis was performed as previously described $(21,22)$. Briefly, the cells were lysed in lysis buffer [ $25 \mathrm{mM}$ HEPES (pH 7.7), $400 \mathrm{mM} \mathrm{NaCl}, 0.5 \%$ Triton X-100, $1.5 \mathrm{mM} \mathrm{MgCl}_{2}, 2 \mathrm{mM}$ EDTA, $2 \mathrm{mM}$ DTT, $0.1 \mathrm{mM}$ phenylmethylsulfonyl fluoride, protease inhibitors (10 $\mu \mathrm{g} / \mathrm{ml}$ leupeptin, $2 \mu \mathrm{g} / \mathrm{ml}$ pepstatin, $50 \mu \mathrm{g} / \mathrm{ml}$ antipain, $1 \mathrm{mM}$ benzamidine, $2 \mu \mathrm{g} / \mathrm{ml}$ aprotinin, $20 \mu \mathrm{g} / \mathrm{ml}$ chymostain), and phosphatase inhibitors $\left(50 \mathrm{mM} \mathrm{NaF}, 1 \mathrm{mM} \mathrm{Na}_{3} \mathrm{VO}_{4}\right.$, $20 \mathrm{mM} \beta$-GP glycerophosphate, $25 \mathrm{mM}$ p-Nitrophenyl phosphate disodium)], and centrifuged at 14,000 $\mathrm{x} g$ for $10 \mathrm{~min}$ at $4^{\circ} \mathrm{C}$. The supernatant was removed and the protein determination was using BCA method, $30 \mu \mathrm{g}$ of protein was run on an $10 \%$ SDS-polyacrylamide gel (PAGE), then transferred onto nitrocellulose polyvinylidene difluoride membranes, blocked with 5\% non-fat milk (NFM), for $1 \mathrm{~h}$ at room temperature and probed with primary polyclonal antibodies (anti-c-Myc, mouse anti-human, 1:1,000 dilution, Cat. no. 5605S; anti-E-cadherin, rabbit anti-human, 1:1,000 dilution, Cat. no. 14472; anti-matrix metalloproteinase (MMP)-9, rabbit anti-human, 1:1,000 dilution, Cat. no. 13667; all antibodies were obtained from Cell Signaling Technology, Danvers, MA, USA). Secondary antibodies were used goat HRP anti-rabbit (1:5,000 dilution, 
Cat. no. 5160-2504, Bio-Rad Laboratories, Inc., Hercules, CA, USA) and goat HRP anti-mouse (1:5,000 dilution, Cat. no. STAR207P, Bio-Rad Laboratories, Inc.). The blots were detected by an enhanced chemiluminescence (ECL) system (Thermo Fisher Scientific, Inc.), and developed using X-ray film. $\beta$-actin (1:5,000 dilution, Cat. no. A2228-200UL, Sigma Chemical Co., St. Louis, MO, USA) served as an internal positive control for all western blots.

RNA extraction. Total RNA from the FFPE specimens was extracted using the RecoverAll ${ }^{\mathrm{TM}}$ Total Nucleic Acid Isolation kit (Thermo Fisher Scientific, Inc.) according to the manufacturer's instructions. Total RNA from the cells was collected using TRIzol reagent (Ambion; Thermo Fisher Scientific, Inc.).

Reverse transcription-quantitative PCR (RT-qPCR). RT-qPCR was performed to detect the hsa-miR-24 and c-Myc mRNA levels. To detect mature hsa-miR-24, we performed reverse transcription to convert the RNA into cDNA using the TaqMan miRNA Reverse Transcription kit (Applied Biosystems Inc., Foster City, CA, USA). Polymerase chain reaction mixtures containing TaqMan Universal Master Mix II (hsa-miR-24) and TaqMan miRNA and gene expression assays were performed according to the manufacturer's instructions (gradient S, Mastercycler, Eppendorf, Germany). To isolate c-Myc mRNA, total RNA was extracted from the cells using TRIzol reagent and reverse transcribed using a high capacity RNA-cDNA kit (Applied Biosystems Inc.). cDNA was quantified on an ABI Prism 7900 sequence detection system (Applied Biosystems Inc.). PCR was performed using Power SYBR-Green PCR master mix (Applied Biosystems Inc.). The cycling parameters were as follows: $50^{\circ} \mathrm{C}$ for $2 \mathrm{~min}$ and $95^{\circ} \mathrm{C}$ for $10 \mathrm{~min}$ followed by 40 cycles at $95^{\circ} \mathrm{C}$ for $15 \mathrm{sec}$ and annealing/extension at $60^{\circ} \mathrm{C}$ for $1 \mathrm{~min}$. U6 snRNA or glyceraldehyde 3-phosphate dehydrogenase (GAPDH) were used to normalize the hsa-miR-24 and c-Myc values, respectively. Data were analyzed by the $2^{-\Delta \Delta \mathrm{Cq}}$ method to determine relative changes in miRNA and gene expression (23). The primers for hsa-miR-24 were reported in our previous study (17). The primers for c-Myc PCR amplification were as follows: Forward, AAAGGCCCCCAAGGTAGTTA and reverse, GCACAAGAGTTCCGTAGCTG.

Dual-luciferase reporter assay. The luciferase plasmids, Psi-check2-c-MYC-3'UTR and Mutant Psi-check2-c-MYC-3'UTR (gifts from Dr J. Lieberman, Harvard Medical School, Boston, MA, USA), were transiently transfected with hsa-miR-24 mimic into 5-8F cells as previously described (23). In brief, the cells were seeded into 24-well plates and co-transfected $24 \mathrm{~h}$ later with pMiR-3'-UTR, $30 \mathrm{nmol} / 1$ hsa-miR-24 mimic, or miR control and a pRL plasmid control using Plus reagent (Invitrogen) according to the manufacturer's instructions. After $48 \mathrm{~h}$, the luciferase activities were detected using a Dual-Luciferase reporter system (Promega, Madison, WI, USA). All experiments were performed in triplicate, and the results are presented as the means of these independent experiments.

c-Myc analysis from RPPA protein profiling. A heatmap of the proteins tested by RPPA was generated using the Cluster 3.0 software program, and the results were visualized using the TreeView 1.6 software program as previously described (20). Pathway analysis was performed using Ingenuity Pathway Analysis (Qiagen, Hilden, Germany), as previously described $(20,24)$.

Statistical analysis. Statistical analysis was performed using SPSS software (version 16.0, IBM Corp., Armonk, NY, USA). Statistical analyses were performed by one-way analysis of variance (ANOVA) when there were more than 2 groups. The Bonferroni correction method of multiple comparisons with parental and control vector groups was used when the probability for ANOVA was statistically significant. The Student's t-test was used when there were only 2 groups. Actuarial survival rates were calculated using the Kaplan-Meier method, and differences in survival in univariate comparisons were compared using the log-rank test. Data are expressed as the means $\pm \mathrm{SD}$, and P-values $<0.05$ were considered to indicate statistically significant differences.

\section{Results}

hsa-miR-24 is a negative metastasis-related prognostic factor in NPC. To determine whether miR-24 is involved in tumor progression and metastasis, a RT-qPCR analysis was performed to detect its expression levels. We found that hsa-miR-24 expression was lower in the metastatic NPC tissue $(n=11)$, than in the non-metastatic tissue $(n=12)$ (Fig. 1A). Kaplan-Meier survival analysis suggested that a high expression of hsa-miR-24 was likely associated with a longer progression-free survival and metastasis-free survival (Fig. 1B and C). Due to the small sample size in the training group, the P-value was not statistically significant (Fig. $1 \mathrm{~B}, \mathrm{P}=0.381$; Fig. $1 \mathrm{C}, \mathrm{P}=0.240$ ); in the validation group, however, the prognostic value of hsa-miR-24 was statistically significant (Fig. 1D, P=0.039). Moreover, nCounter human miR assay profiling analysis revealed that the hsa-miR-24 expression level in the NPC metastatic tissue was significantly lower than that in the non-metastatic tissue in the validation group as expected, (Fig. 1D, $n=60$, $\mathrm{P}=0.039$ ), and survival analysis further indicated that the hsa-miR-24 expression level was negatively associated with distant metastasis (Fig. $1 \mathrm{E}, \mathrm{n}=60, \mathrm{P}=0.037$ ). The clinical significance suggests that hsa-miR-24 may also serve as an effective predictive biomarker in NPC.

hsa-miR-24 inhibits proliferation in vitro. To better elucidate the role of hsa-miR-24 in NPC, we established a cell line stably expressing hsa-miR-24, namely the C666-1 cells and named this cell line C666-1-miR-24, using lentiviral transfection. RT-qPCR analysis indicated that hsa-miR-24 was increased 5-7 fold in the stably transfected cells compared to the miR-control group (Fig. 2A). The RFP image of hsa-miR-24 also supported the result of RT-qPCR (Fig. 2B). Subsequently, using PrestoBlue assay and colony formation assay, we found that cell viability and cell growth ability were suppressed in the hsa-miR-24 stably transfected cells compared with the miR-control group (Fig. $2 \mathrm{C}$ and $\mathrm{D}, \mathrm{P}<0.05$ and $\mathrm{P}<0.01$ ). The above-mentioned results indicated that the overexpression of hsa-miR-24 inhibited cell growth. 

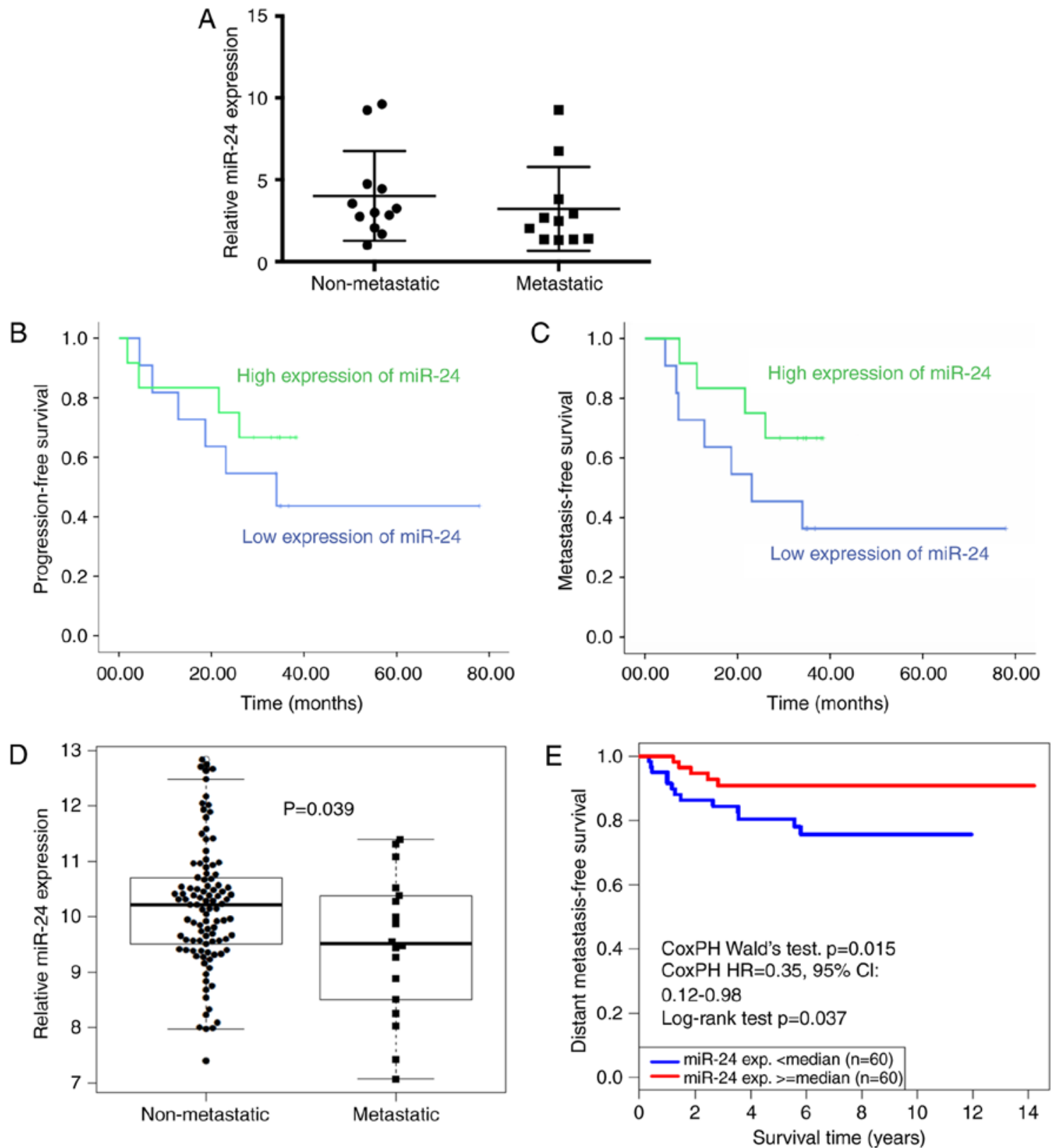

Figure 1. hsa-miR-24 is a negative metastasis-related prognostic factor in NPC. (A) The hsa-miR-24 expression level in metastatic and non-metastatic NPC tissue in the training group was examined by RT-qPCR. (B and C) Kaplan-Meier analysis of progression-free survival and distant metastasis-free survival according to hsa-miR-24 expression in the training group [(B) $\mathrm{P}=0.381$; (C) $\mathrm{P}=0.240]$. (D) Relative hsa-miR-24 expression level in NPC tissue in the validation group $(\mathrm{n}=60, \mathrm{P}=0.039)$. Raw data were analyzed with $2^{-\Delta \Delta \mathrm{Cq}}$. U6 snRNA was used as an internal control. (E) Kaplan-Meier analysis of distant metastasis-free survival according to hsa-miR-24 expression in the validation group ( $\mathrm{n}=60, \mathrm{P}=0.037)$. NPC, nasopharyngeal carcinoma.

hsa-miR-24 inhibits NPC cell migration and invasion. To further investigate the biological functions of hsa-miR-24, we examined the cells migratory and invasive ability by performing wound-healing assay and invasion assay. Our results revealed that the hsa-miR-24 stably transfected cell line ( C666-1-miR-24) migrated at a significantly slower rate compared with the miR-control (Fig. 3A, P<0.01). Transwell invasion assay revealed that the C666-1-miR-24 cells were less likely to invade the lower chamber, and the cell invasion rate was 50\% lower compared with that of the miR-control cells (Fig. 3B, $\mathrm{P}<0.01$ ). These results suggested that a high hsa-miR-24 level suppresses NPC cell migration and invasion.

c-Myc is a direct target of hsa-miR-24 in the regulation of NPC metastasis. To clarify the molecular mechanisms behind the regulatory effects of hsa-miR-24 on NPC cell proliferation and metastasis, we searched for hsa-miR-24 potential candidate targets in the regulation of NPC metastasis. A previous study reported that c-Myc was as a potential target of hsa-miR-24; within its 3'-UTR, there are hsa-miR-24 'seedless' binding sites (25). In this study, to validate whether has-miR-24 binds to the 3'UTR of c-Myc in NPC, we conducted luciferase reporter assay to determine whether c-Myc was a functional target of hsa-miR-24. Increasing the hsa-miR-24 expression level markedly reduced the luciferase activity in the $5-8 \mathrm{~F}$ cells transfected with pMiR-c-Myc-3'-UTR compared with the miR-control (approximately 50\%, Fig. 4A), while the overexpression of hsa-miR-24 had no effect on the mutant c-Myc 3'-UTR. Furthermore, the results of RT-qPCR and western blot 
A

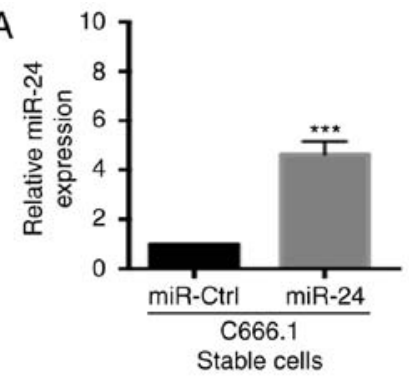

C

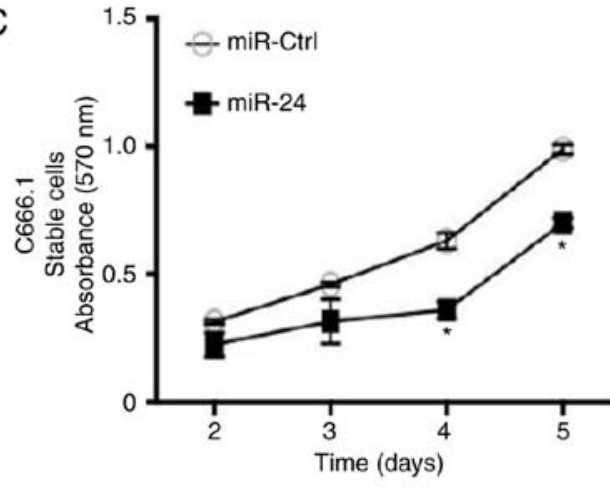

B

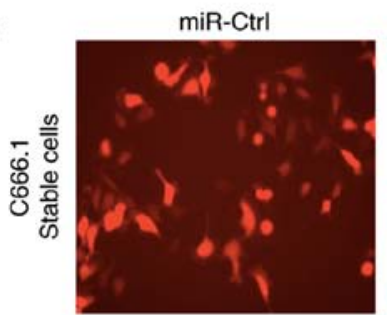

D
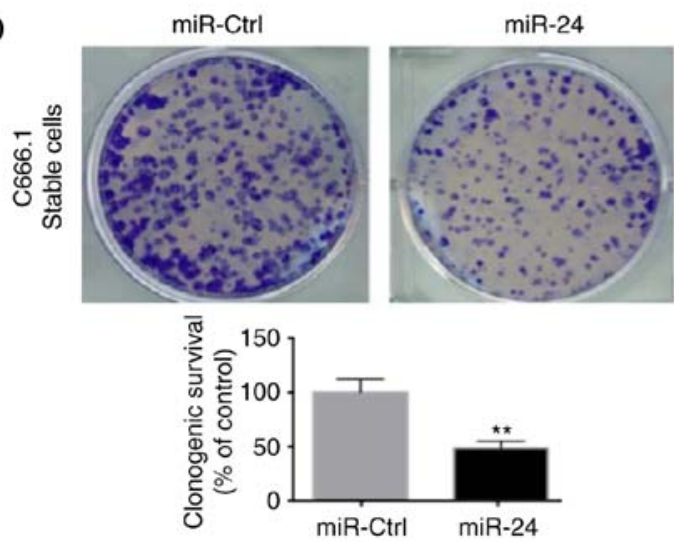

Figure 2. hsa-miR-24 inhibits proliferation in vitro. (A) Relative expression of hsa-miR-24 in the C666-1-miR-24 cells compared with the miR-control cells examined by RT-qPCR, ${ }^{* * *} \mathrm{P}<0.001$. (B) hsa-miR-24 expression examined by RFP detection in C666-1-miR-24 and C666-1-miR-control cells. (C) Impaired viability of C666-1-miR-24 cells compared with miR-control as measured by Presto Blue assay, ${ }^{*} \mathrm{P}<0.05$. (D) Proliferative ability of the C666-1-miR-24 cells compared with miR-control, ${ }^{* *} \mathrm{P}<0.01$. Data represent the means \pm SD of 3 independent experiments; P-values were calculated with the Student's t-Test.

A
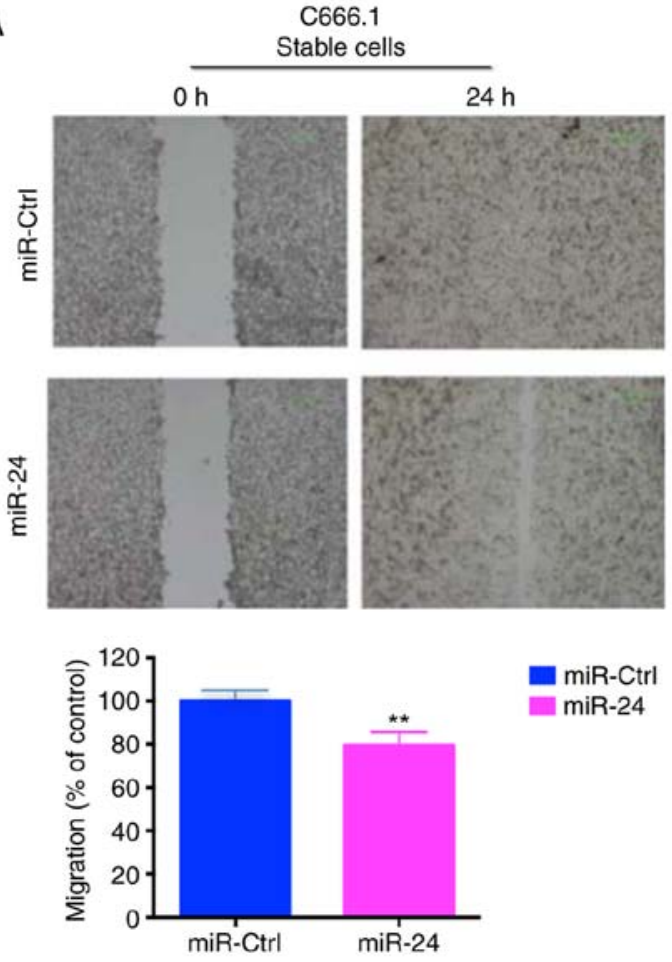
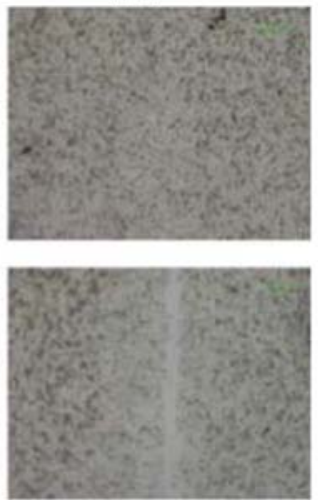

miR-Ctrl
B
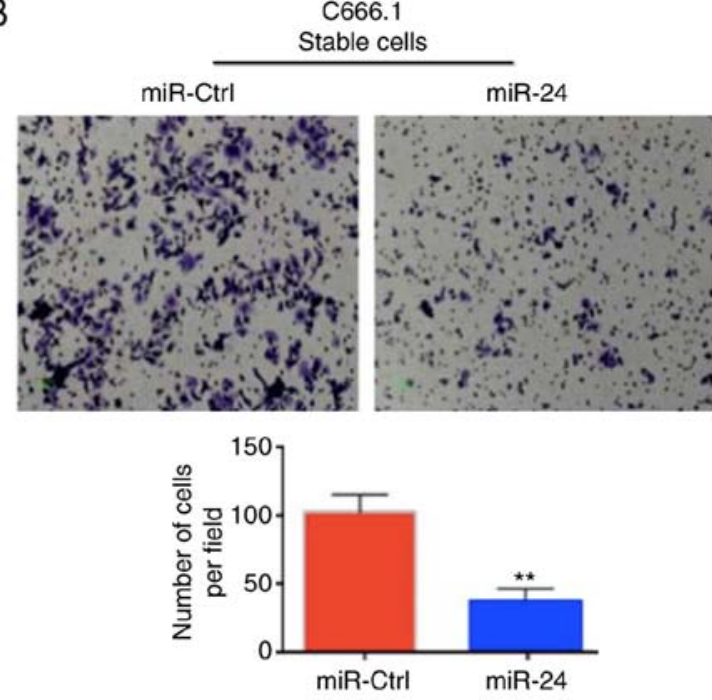

Figure 3. hsa-miR-24 inhibits NPC cell migration and invasion. (A) Cell migratory ability was significantly inhibited by the stable overexpression of has-miR-24 (C666-1-miR-24 cells), compared with the miR-control, ${ }^{* *} \mathrm{P}<0.01$. (B) Invasive ability of C666-1-miR-24 cells compared with the miR-control by examined by Transwell invasion assay, ${ }^{* *} \mathrm{P}<0.01$. Data represent the means \pm SD from 3 independent experiments; P-values were calculated with the Student's t-test. NPC, nasopharyngeal carcinoma.

analysis verified that the c-Myc mRNA and protein levels were significantly lower in the cells stably expressing hsa-miR-24
(C666-1-miR-24 cells) compared with the miR-control cells (4-5-fold, Fig. 4B and C, respectively). Transfection of the 

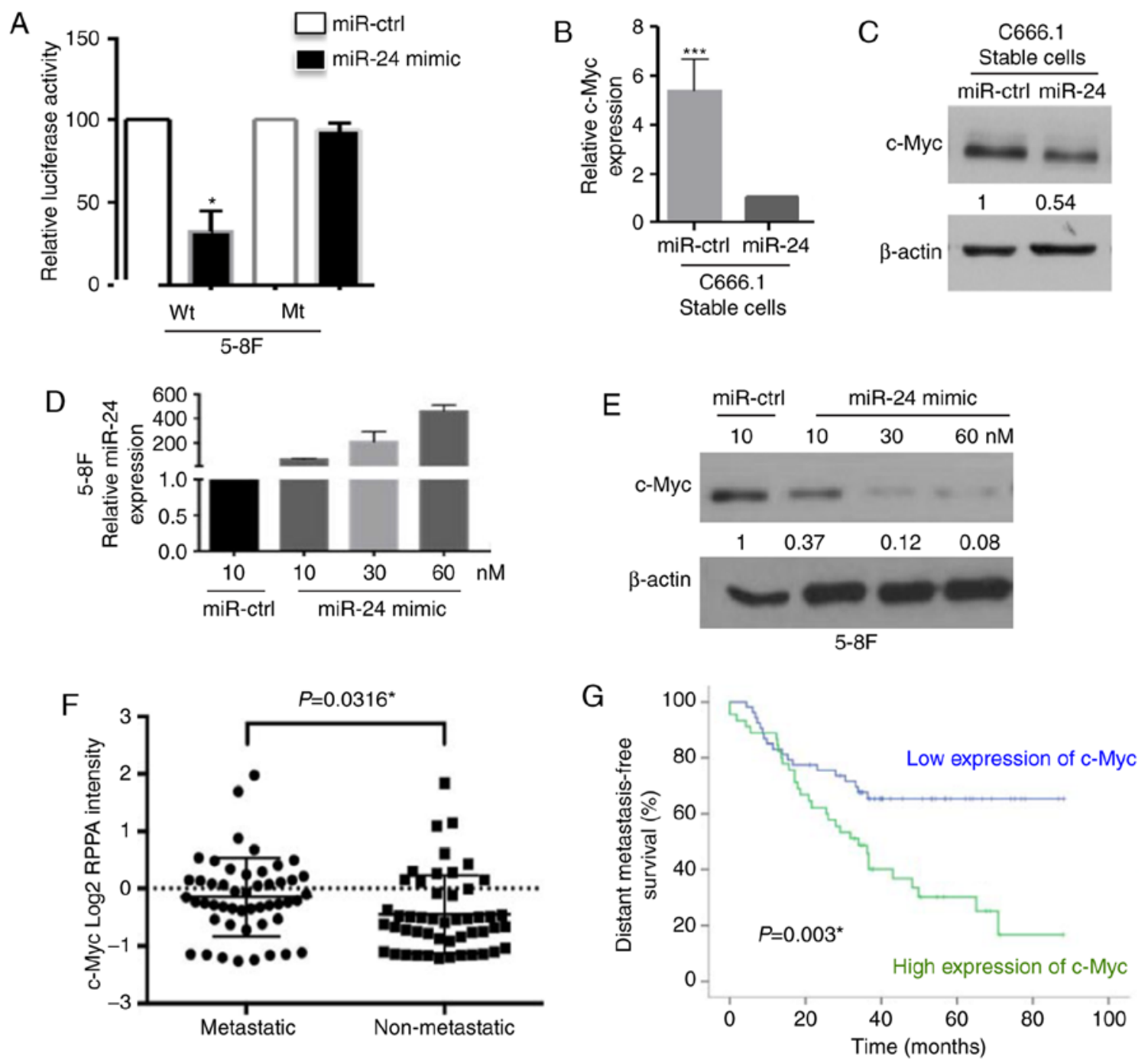

Figure 4. c-Myc is a direct target of hsa-miR-24 in regulating NPC metastasis. (A) Relative luciferase activity of miR luciferase constructs containing the 3'-UTR of c-Myc wild-type (Wt) and mutant c-Myc 3'-UTR (Mt), co-transfected with miR-24 mimics or miRNA control into 5-8F cells. Data represent the means \pm SD of 3 independent experiments and were normalized to the control; ${ }^{*} \mathrm{P}<0.05$. (B) Quantification of c-Myc expression in C666-1-miR-24 cells compared with the miR-control by RT-qPCR, ${ }^{* * *} \mathrm{P}<0.001$. (C) c-Myc protein expression in C666-1-miR-24 cells compared with miR-control examined by western blot analysis. The relative protein expression level was normalized to $\beta$-actin. (D and E) hsa-miR-24 expression and c-Myc protein expression in $5-8 \mathrm{~F}$ cells treated with increasing amount of miR-24 mimic and mimic control. The relative protein expression level was normalized to $\beta$-Aatin. (F) RPPA proteomic profiling comparing the c-Myc protein expression level between metastatic and non-metastatic NPC samples $(\mathrm{n}=49, \mathrm{P}=0.0316)$. (G) Kaplan-Meier analysis of long-term metastasis-free survival in samples with a differential c-Myc expression level $(\mathrm{n}=49, \mathrm{P}=0.003)$. Data represent the means \pm SD of 3 independent experiments; P-values were calculated with the Student's t-test. NPC, nasopharyngeal carcinoma.

5-8F cells with hsa-miR-24 mimic led to a markedly increased hsa-miR-24 expression level and a decreased c-Myc protein level in a dose-dependent manner (Fig. 4D and E), indicating that hsa-miR-24 negatively regulates c-Myc expression.

Subsequently, we exploited the RPPA proteomic profiling of metastatic and non-metastatic NPC specimens from our previous study (20). Protein expression analysis revealed that c-Myc expression was higher in the metastatic NPC tumors compared with the non-metastatic NPC tumors (Fig. 4F), $(\mathrm{n}=49, \mathrm{P}=0.0316)$. Kaplan-Meier analysis indicated that a low c-Myc expression was associated with the long-term metastasis-free survival rate, while a high c-Myc expression was associated with a poor prognostic outcome in NPC (Fig. 4G), $(\mathrm{n}=49, \mathrm{P}=0.003)$.

In addition, we examined the influence of hsa-miR-24 and c-Myc on cancer survival using an miRNA and gene survival analysis platform (SurvMicro and SurvExpres, data not shown). We found that high levels of hsa-miR-24 were associated with a better overall survival in head and neck squamous cell carcinoma, and that a high expression of hsa-miR-24 represented a low prognostic risk. In addition, high levels of c-Myc were consistent with a poor overall survival for head and neck squamous cell carcinoma, and a high expression of c-Myc represented a high prognostic risk (data not shown). These data supported our above-mentioned findings. Taken together, hsa-miR-24 is an anti-metastatic factor, can directly target c-Myc-3' UTR, negatively regulate c-Myc mRNA and protein expression; c-Myc is a pro-metastatic risk factor in NPC.

hsa-miR-24 suppresses NPC metastasis by regulating the $c-M y c / E M T$ axis. To further elucidate the signaling pathway 
A

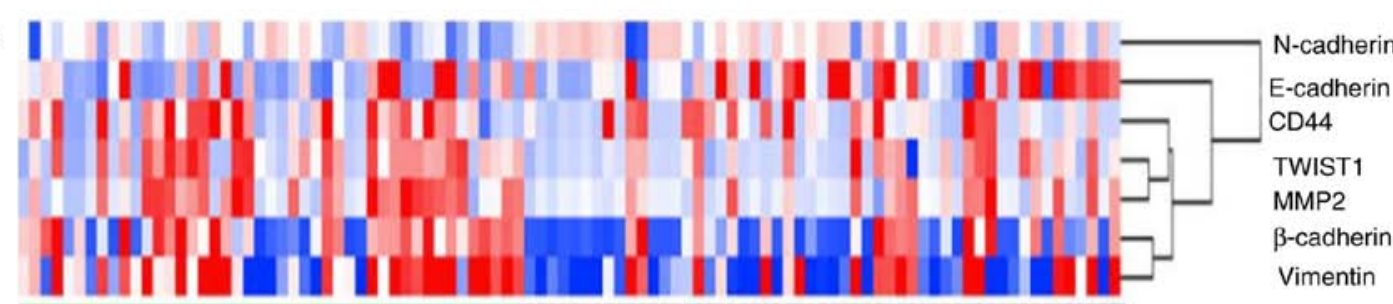
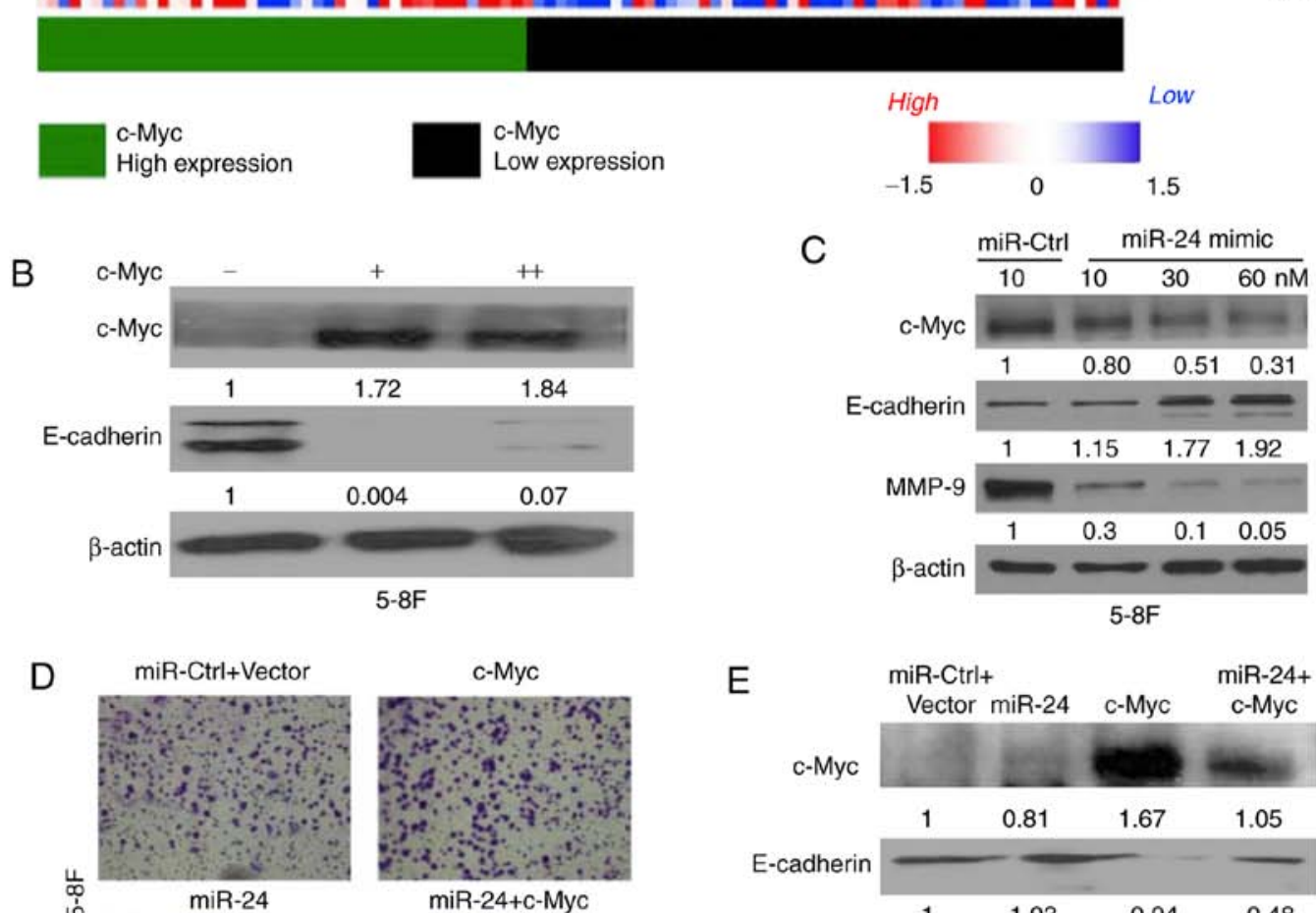

E

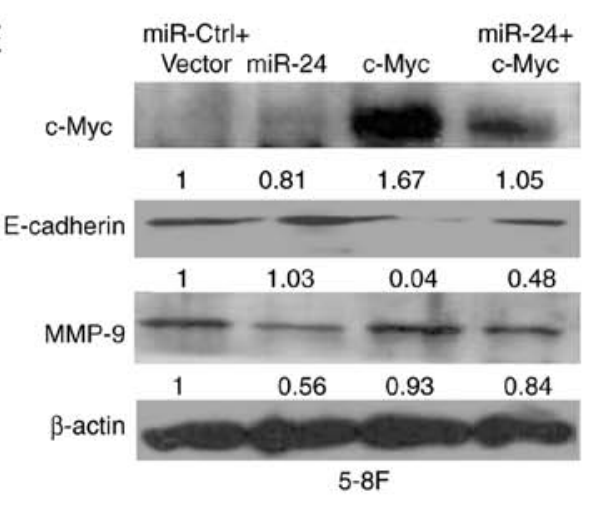

Figure 5. hsa-miR-24 suppresses NPC metastasis by regulating the c-Myc/EMT axis. (A) Signaling pathway analysis of pathways related with differential c-Myc expression from RPPA proteomic profiling. (B) Suppression of E-cadherin levels by c-Myc expression (or vector control) in 5-8F cells. Relative expression was normalized to $\beta$-actin. (C) miR-24 suppresses c-Myc and MMP-9 levels and increases E-cadherin levels. The relative expression was normalized to $\beta$-actin. (D) Invasive ability of 5-8F cells transfected with c-Myc alone, hsa-miR-24 mimic alone, or co-transfected miR-24 mimic and c-Myc, miR-control and vector control, ${ }^{*} \mathrm{P}<0.05,{ }^{* *} \mathrm{P}<0.01,{ }^{* * *} \mathrm{P}<0.001$. (E) c-Myc, E-cadherin and MMP-9 levels in 5-8F cells transfected with c-Myc alone, hsa-miR-24 mimic alone, or co-transfected miR-24 mimic and c-Myc, miR-control and vector control. The relative expression was normalized to $\beta$-actin. Data represent the means \pm SD of 3 independent experiments; P-values were calculated with one-way analysis of variance (ANOVA). NPC, nasopharyngeal carcinoma; MMP, matrix metalloproteinase.

involved in the effects of hsa-miR-24 on NPC metastasis through the targeting of c-Myc, first, we performed Ingenuity Pathway analysis to examine the RPPA profiling obtained. We found that a high expression of c-Myc was associated with a high expression of EMT mesenchymal markers, such as N-cadherin, TWIST, MMP2 and Vimentin, while the epithelial marker, E-cadherin, was negatively associated with high c-Myc (Fig. 5A). The signaling heatmap indicated that a high
c-Myc expression promoted NPC metastasis by inducing EMT. Indeed, the overexpression of c-Myc markedly suppressed the E-cadherin expression level (Fig. 5B).

We then upregulated hsa-miR-24 expression to determine whether it could further regulate EMT. We found that the inhibition of c-Myc expression by transfection with hsa-miR-24 mimic was accompanied by an increase in E-cadherin expression and a decrease in MMP-9 expression in the 5-8F cells 
(Fig. 5C). Moreover, the cell invasive ability was increased by c-Myc overexpression (Fig. 5D), while co-transfection with the c-Myc expression plasmind and hsa-miR-24 mimic abrogated this effect (Fig. 5D), and the expression of E-cadherin was increased again (Fig. 5E). On the whole, our results indicate that hsa-miR-24 suppresses NPC cell invasion by regulating the c-Myc/EMT axis.

\section{Discussion}

Distant metastasis has been considered as the major cause of mortality in NPC. The lack of effective treatment strategies for metastatic NPC has led to the low survival rate. Novel approaches for targeting NPC metastasis are urgently required. Emerging evidence indicates that miRNA-based therapeutics represents an attractive treatment strategy for cancer metastasis (15). In the present study, we found low miR-24 levels in the metastatic NPC tumors, and that high levels of hsa-miR-24 were associated with a longer progression-free and metastasis-free patient survival. hsa-miR-24 suppressed cell migration and invasion by targeting c-Myc 3'-UTR in NPC metastasis. The upregulation of c-Myc expression increased the invasive ability of the NPC cells, while hsa-miR-24 suppressed c-Myc expression, thus inhibiting its ability to induce metastasis. Our findings suggest that the miR-24/c-Myc axis facilitates metastasis via EMT.

Several miRNA signature profiling has been conducted in NPC to predict thje distant metastasis risk and prognostic value in NPC $(19,26,27)$. Bruce et al (19) performed global miR profiling of two independent cohorts and identified an miR signature that was capable of predicting distant metastasis in NPC. This signature may enhance the prognostic value of the current 'gold standard' of TNM classification of malignant tumors to inform treatment options for patients who are at high risk of disease progression.

Our previous studies indicated that hsa-miR-24 was a tumor suppressor and functioned as a radiosensitizer in NPC through the inhibition of CSN5/Jab1 (16-18). In the current study, we observed that hsa-miR-24 expression was lower in the metastatic tissue than in non-metastatic tissue, which also suggests that hsa-miR-24 is a tumor suppressor. To further elucidate the underlying mechanisms, we identified a potential target of hsa-miR-24 in the regulation of cancer metastasis. Using different cancer databases and luciferase reporter assay, we identified the 3'-UTR of c-Myc as a novel target of hsa-miR-24 in the regulation of NPC metastasis.

c-MYC is an oncogene frequently overexpressed in many types of human cancer; it can promote cell proliferation, cell differentiation blockade, gene instability, and tumor invasion and metastasis (28-30). The ONCOMINE database analysis approved that MYC was overexpressed in NPC compare to normal nasopharynx (data not shown). The overexpression and amplification of c-Myc have been closely associated with a poor survival, considered as a predictor of poor prognosis for clinical patients in several types of cancer, such as gastric cancer, colorectal cancer, bladder cancer and breast cancer (31-34). It is thus understandable that c-Myc promotes the migration and invasion of NPC cells $(35,36)$. Our previous RPPA based proteomic profiling suggested that c-Myc was one of the major driver proteins for metastasis risk for NPC (20).
However, its exact mechanisms of action in NPC metastasis were not identified.

Our TCGA analysis (data not shown) revealed that high levels of hsa-miR-24 were associated with a better overall survival and a low prognostic risk, while high levels of c-Myc were consistent with a poor overall survival and a high prognostic risk in head and neck, which further supported our hypothesis that hsa-miR-24 is associated with c-Myc. Indeed, luciferase reporter assay identified that c-Myc was a direct target of hsa-miR-24, which is consistent with the findings of a previous study that within its 3'-UTR, there is a hsa-miR-24 'seedless' binding site (25). Several target genes of miR-24 have been reported, such as MKP-7, ST7L, DHFR, RegIV and Net1 (37-41). All these genes were predicted by miRNA algorithms, of which contain conserved 3'-UTR sequences complementary to a seed region of the hsa-miR-24. Lal et al demonstrated that MYC was a direct target of hsa-miR-24 by using miRNA overexpression methods combined with bioinformatics analysis and miRNA algorithms, which could abate the high error rate and missing key target prediction when using miRNA algorithms alone (25). Our findings are in agreement with those of the study by Lal et al, using the overexpression of hsa-miR-24 and NPC patient metastasis protein profiling prognostic analysis. To date, at least to the best of our knowledge, there is no study available on the mechanisms through which miR-24 regulates c-Myc in promoting NPC metastasis. This study demonstrates for the first time that miR-24 is a c-Myc regulator that can serve as an anti-metastatic factor in NPC. In particular, by using signaling analysis, we found that c-Myc was inversely associated with metastasis-free overall survival and with EMT.

EMT is considered an early and key step in metastasis (42). A hallmark of EMT is the loss of E-cadherin, which plays a core role in cell-to-cell adhesion (43). The loss of E-cadherin expression is often accompanied by an increase in the levels of the mesenchymal and extracellular matrix biomarkers, MMP-2, MMP-9, N-cadherin and vimentin $(44,45)$. EMT is regulated by a variety of signaling pathways, including transforming growth factor (TGF)- $\beta /$ Snail, glycogen synthase kinase (GSK) $3 \beta$, epidermal growth factor receptor (EGFR), Notch and extracellular-signal-regulated kinase (ERK)/mitogen-activated protein kinase (MAPK) (46). It has been reported that c-Myc is critical for maintaining epithelial originated cancer metastasis, which can activate the EMT phenotype with the loss of E-cadherin expression via the TGF- $\beta /$ Snail and RhoA signaling pathways, thereby causing cell invasion and migration $(47,48)$. The findings of this study are in agreement with these findings in that c-Myc a high expression was associated with the loss of E-cadherin expression in NPC. We further confirmed that c-Myc induced cell invasion, with the loss of E-cadherin expression. hsa-miR-24, as a regulator of c-Myc, can abrogate NPC cell invasion promoted by c-Myc overexpression, and is associate with the restoration of E-cadherin expression.

In conclusion, the findings of this study demonstrate that hsa-miR-24 is an anti-metastatic regulator in NPC by regulating the c-Myc/EMT axis; hsa-miR-24 plays a protective role in NPC. Our finding provides a promising miR-target for the prognosis of patients with a high TNM grade and provide novel therapeutic approaches for NPC. 


\section{Acknowledgements}

The authors would like to thank Professor Ernest Martinez (University of California-Riverside, Riverside, CA, USA) for the kind gift of the pCbS-human-c-Myc plasmid, as well as Professor J. Lieberman (Harvard Medical School, Boston, MA, USA) for sharing the Psi-check2-c-MYC-3'UTR (Mutant) and pRL-Luciferase-cMYC-3'UTR plasmids. We would also like to thank Dr Ann Sutton from the Department of Scientific Publications at MD Anderson for editing the manuscript.

\section{Funding}

This study was supported by grants from the National Natural Science Foundation of China (81372410 to HY) and the Scientific and Technological Project of Guangdong, China (2015A020211006 to HY), and grants from the National Cancer Institute (R01 CA90853 to FXC) and the Sister Institution Network Fund (FXC). The University of Texas MD Anderson Cancer Center is supported in part by the National Institutes of Health through Cancer Center Support Grant P30 CA016672.

\section{Availability of data and materials}

The datasets used and/or analyzed during the current study are available from the corresponding author on reasonable request.

\section{Authors' contributions}

$\mathrm{BS}, \mathrm{TX}$ and $\mathrm{FXC}$ were involved in the conception and design of the study. TX, BS and FXC were involved in the development of the methodology. JPB, KWY, NZ, ZH, GZ, JL, FFL, HY and FXC were involved in the acquisition of the data (acquired and managed patient data, provided facilities, e.t.c.). BS, TX, JPB, KWY, FFL and FXC were involved in the analysis and interpretation of the data (e.g., statistical analysis, biostatistics and computational analysis). BS, TX and $\mathrm{FXC}$ were involved in the writing, reviewing and/or revision of the manuscript. BS, TX, HY and FXC were involved in administrative, technical or material support (i.e., reporting or organizing data, constructing databases). FXC supervised the study. All authors have read and approved the final manuscript.

\section{Ethics approval and consent to participate.}

The Ethics Committee of the First People's Hospital of Foshan, Foshan, China, approved the study protocol. All patients provided written informed consent pior to participation in this study.

\section{Patient consent for publication}

Not applicable.

\section{Competing interests}

The authors declare that they no competing interests.

\section{References}

1. Tsao SW, Yip YL, Tsang CM, Pang PS, Lau VM, Zhang G and Lo KW: Etiological factors of nasopharyngeal carcinoma. Oral Oncol 50: 330-338, 2014.

2. Chua MLK, Wee JTS, Hui EP and Chan ATC: Nasopharyngeal carcinoma. Lancet 387: 1012-1024, 2016.

3. Chan A, Grégoire V, Lefebvre JL, Licitra L, Felip E and group EEEgw: Nasopharyngeal Cancer: EHNS-ESMO-ESTRO clinical practice guidelines for diagnosis, treatment and follow-up. Ann Oncol 21: v187-v189, 2010.

4. Blanchard P, Lee A, Marguet S, Leclercq J, Ng WT, Ma J, Chan AT, Huang PY, Benhamou E, Zhu G, et al: Chemotherapy and radiotherapy in nasopharyngeal carcinoma: An update of the MAC-NPC meta-analysis. Lancet Oncol 16: 645-655, 2015.

5. Huang PY, Zeng Q, Cao KJ, Guo X, Guo L, Mo HY, Wu PH, Qian CN, Mai HQ and Hong MH: Ten-year outcomes of a randomised trial for locoregionally advanced nasopharyngeal carcinoma: A single-institution experience from an endemic area. Eur J Cancer 51: 1760-1770, 2015.

6. Lee AW, Ma BB, Ng WT and Chan AT: Management of nasopharyngeal carcinoma: Current practice and future perspective. J Clin Oncol 33: 3356-3364, 2015.

7. Lin S and Gregory RI: MicroRNA biogenesis pathways in cancer. Nat Rev Cancer 15: 321-333, 2015.

8. Calin GA and Croce CM: MicroRNA signatures in human cancers. Nat Rev Cancer 6: 857-866, 2006.

9. Klein U, Lia M, Crespo M, Siegel R, Shen Q, Mo TW, Ambesi-Impiombato A, Califano A, Migliazza A, Bhagat G, et al: The DLEU2/miR-15a/16-1 cluster controls b cell proliferation and its deletion leads to chronic lymphocytic leukemia. Cancer Cell 17: 28-40, 2010.

10. Calin GA, Dumitru CD, Shimizu M, Bichi R, Zupo S, Noch E, Aldler H, Rattan S, Keating M, Rai K, et al: Frequent deletions and down-regulation of micro-RNA genes miR15 and miR16 at $13 q 14$ in chronic lymphocytic leukemia. Proc Natl Acad Sci USA 99: 15524-15529, 2002.

11. Liu G: Augmentation of response to chemotherapy by microRNA-506 through regulation of RAD51 in serous ovarian cancers. J Natl Cancer Inst 109: 2017.

12. Ding X, Park SI, McCauley LK and Wang CY: Signaling between transforming growth factor $\beta$ (TGF- $\beta$ ) and transcription factor SNAI2 represses expression of microRNA miR-203 to promote epithelial-mesenchymal transition and tumor metastasis. J Biol Chem 288: 10241-10253, 2013.

13. Ma L, Young J, Prabhala H, Pan E, Mestdagh P, Muth D, Teruya-Feldstein J, Reinhardt F, Onder TT, Valastyan S, et al: miR-9, a MYC/MYCN-activated microRNA, regulates E-cadherin and cancer metastasis. Nat Cell Biol 12: 247-256, 2010.

14. Asangani IA, Rasheed SA, Nikolova DA, Leupold JH, Colburn NH, Post S and Allgayer H: MicroRNA-21 (miR-21) post-transcriptionally downregulates tumor suppressor Pdcd4 and stimulates invasion, intravasation and metastasis in colorectal cancer. Oncogene 27: 2128-2136, 2008.

15. Ma L, Reinhardt F, Pan E, Soutschek J, Bhat B, Marcusson EG, Teruya-Feldstein J, Bell GW and Weinberg RA: Therapeutic silencing of miR-10b inhibits metastasis in a mouse mammary tumor model. Nat Biotechnol 28: 341-347, 2010.

16. Wang S, Zhang R, Claret FX and Yang $\mathrm{H}$ : Involvement of microRNA-24 and DNA methylation in resistance of nasopharyngeal carcinoma to ionizing radiation. Mol Cancer Ther 13: 3163-3174, 2014.

17. Wang S, Pan Y,Zhang R, Xu T, Wu W, Zhang R, Wang C, Huang H, Calin CA, Yang $\mathrm{H}$ and Claret FX: Hsa-miR-24-3p increases nasopharyngeal carcinoma radiosensitivity by targeting both the 3'UTR and 5'UTR of Jab1/CSN5. Oncogene 35: 6096-6108, 2016.

18. Claret FX, Hibi M, Dhut S, Toda T and Karin M: A new group of conserved coactivators that increase the specificity of AP-1 transcription factors. Nature 383: 453-457, 1996.

19. Bruce JP, Hui AB, Shi W, Perez-Ordonez B, Weinreb I, Xu W, Haibe-Kains B, Waggott DM, Boutros PC, O'Sullivan B, et al: Identification of a microRNA signature associated with risk of distant metastasis in nasopharyngeal carcinoma. Oncotarget 6: 4537-4550, 2015

20. Xu T, Su B, Huang P, Wei W, Deng Y, Sehgal V, Wang D, Jiang J, Zhang G, Li A, et al: Novel biomarkers of nasopharyngeal carcinoma metastasis risk identified by reverse phase protein array based tumor profiling with consideration of plasma Epstein-Barr virus DNA load. Proteomics Clin Appl 11, 2017. 
21. Rassidakis GZ, Claret FX, Lai R, Zhang Q, Sarris AH, McDonnell TJ and Medeiros LJ: Expression of p27(Kip1) and c-Jun activation binding protein 1 are inversely correlated in systemic anaplastic large cell lymphoma. Clin Cancer Res 9: $1121-1128,2003$

22. Kouvaraki MA, Rassidakis GZ, Tian L, Kumar R, Kittas C and Claret FX: Jun activation domain-binding protein 1 expression in breast cancer inversely correlates with the cell cycle inhibitor p27(Kip1). Cancer Res 63: 2977-2981, 2003.

23. Livak KJ and Schmittgen TD: Analysis of relative gene expression data using real-time quantitative PCR and the 2(-Delta Delta C(T)) method. Methods 25: 402-408, 2001.

24. Shull AY, Noonepalle SK, Awan FT, Liu J, Pei L, Bollag RJ, Salman H, Ding ZY and Shi HD: RPPA-based protein profiling reveals eIF4G overexpression and 4E-BP1 serine 65 phosphorylation as molecular events that correspond with a pro-survival phenotype in chronic lymphocytic leukemia. Oncotarget 6 : 14632-14645, 2015.

25. Lal A, Navarro F, Maher CA, Maliszewski LE, Yan N, O'Day E, Chowdhury D, Dykxhoorn DM, Tsai P, Hoffman O, et al: miR-24 Inhibits cell proliferation by targeting E2F2, MYC, and other cell-cycle genes via binding to 'Seedless' 3' UTR MicroRNA recognition elements. Mol Cell 35: 610-625, 2009.

26. Liu N, Chen NY, Cui RX, Li WF, Li Y, Wei RR, Zhang MY, Sun Y, Huang BJ, Chen M, et al: Prognostic value of a microRNA signature in nasopharyngeal carcinoma: A microRNA expression analysis. Lancet Oncol 13: 633-641, 2012.

27. Liu N, Cui RX, Sun Y, Guo R, Mao YP, Tang LL, Jiang W, Liu X, Cheng YK, He QM, et al: A four-miRNA signature identified from genome-wide serum miRNA profiling predicts survival in patients with nasopharyngeal carcinoma. Int J Cancer 134 1359-1368, 2014

28. Boxer LM and Dang CV: Translocations involving c-myc and c-myc function. Oncogene 20: 5595-5610, 2001.

29. Vita M and Henriksson M: The Myc oncoprotein as a therapeutic target for human cancer. Semin Cancer Biol 16: 318-330, 2006.

30. Albihn A, Johnsen JI and Henriksson MA: MYC in oncogenesis and as a target for cancer therapies. Adv Cancer Res 107: 163-224, 2010.

31. Wang X, Liu Y, Shao D, Qian Z, Dong Z, Sun Y, Xing X, Cheng X, Du H, Hu Y, et al: Recurrent amplification of MYC and TNFRSF11B in 8q24 is associated with poor survival in patients with gastric cancer. Gastric Cancer 19: 116-127, 2016.

32. Wang W, Deng J, Wang Q, Yao Q, Chen W, Tan Y, Ge Z, Zhou J and Zhou Y: Synergistic role of Cull and c-Myc: Prognostic and predictive biomarkers in colorectal cancer. Oncol Rep 38: 245-252, 2017

33. Seo HK, Ahn KO, Jung NR, Shin JS, Park WS, Lee KH, Lee SJ and Jeong KC: Antitumor activity of the c-Myc inhibitor KSI-3716 in gemcitabine-resistant bladder cancer. Oncotarget 5: 326-337, 2014

34. Carey JP, Karakas C, Bui T, Chen X, Vijayaraghavan S, Zhao Y, Wang J, Mikule K, Litton JK, Hunt KK and Keyomarsi K: Synthetic lethality of PARP inhibitors in combination with MYC blockade is independent of BRCA status in triple negative breast cancer. Cancer Res 78: 742-757, 2017.

35. Wang WJ, Wu SP, Liu JB, Shi YS, Huang X, Zhang QB and Yao KT: MYC regulation of CHK1 and CHK2 promotes radioresistance in a stem cell-like population of nasopharyngeal carcinoma cells. Cancer Res 73: 1219-1231, 2013.
36. Zhou W, Feng X, Ren C, Jiang X, Liu W, Huang W, Liu Z, Li Z, Zeng L, Wang L, et al: Over-expression of BCAT1, a c-Myc target gene, induces cell proliferation, migration and invasion in nasopharyngeal carcinoma. Mol Cancer 12: 53, 2013.

37. Zaidi SK, Dowdy CR, Van Wijnen AJ, Lian JB, Raza A, Stein JL, Croce CM and Stein GS: Altered Runx1 subnuclear targeting enhances myeloid cell proliferation and blocks differentiation by activating a miR-24/MKP-7/MAPK network. Cancer Res 69 : 8249-8255, 2009.

38. Chen L, Zhang A, Li Y, Zhang K, Han L, Du W, Yan W, Li R, Wang Y, Wang K, et al: MiR-24 regulates the proliferation and invasion of glioma by ST7L via $\beta$-catenin/Tcf- 4 signaling. Cancer Lett 329: 174-180, 2013.

39. Mishra PJ, Song B, Mishra PJ, Wang Y,Humeniuk R, Banerjee D, Merlino G, Ju JF and Bertino JR: MiR-24 tumor suppressor activity is regulated independent of p53 and through a target site polymorphism. Plos One 4: e8445, 2009.

40. Duan Y, Hu L, Liu B, Yu B, Li J, Yan M, Yu Y, Li C, Su L, Zhu Z, et al: Tumor suppressor miR-24 restrains gastric cancer progression by downregulating RegIV. Mol Cancer 13: 127, 2014

41. Papadimitriou E, Vasilaki E, Vorvis C, Iliopoulos D, Moustakas A, Kardassis D and Stournaras C: Differential regulation of the two RhoA-specific GEF isoforms Net1/Net1A by TGF- $\beta$ and miR-24: Role in epithelial-to-mesenchymal transition. Oncogene 31: 2862-2875, 2012

42. Thiery JP: Epithelial-mesenchymal transitions in tumour progression. Nat Rev Cancer 2: 442-454, 2002.

43. Onder TT, Gupta PB, Mani SA, Yang J, Lander ES and Weinberg RA: Loss of E-cadherin promotes metastasis via multiple downstream transcriptional pathways. Cancer Res 68: 3645-3654, 2008.

44. Plaks V, Chou J, Maynard C, Nguyen NH, Kong NW, Solomonov I, Talmi-Frank D, Bonnans C, Sagi I and Werb Z: Targeting matrix metalloproteinases (MMP) for anti-metastatic therapy: Blocking active MMP9 abrogates metastatic niche formation and prevents metastatic seeding in a breast cancer model. Cancer Res 75, 2015.

45. Gravdal K, Halvorsen OJ, Haukaas SA and Akslen LA: A switch from $\mathrm{E}$-cadherin to $\mathrm{N}$-cadherin expression indicates epithelial to mesenchymal transition and is of strong and independent importance for the progress of prostate cancer. Clin Cancer Res 13: 7003-7011, 2007.

46. Gonzalez DM and Medici D: Signaling mechanisms of the epithelial-mesenchymal transition. Sci Signal 7: re8, 2014.

47. Smith AP, Verrecchia A, Fagà G, Doni M, Perna D, Martinato F, Guccione E and Amati B: A positive role for Myc in TGFbeta-induced Snail transcription and epithelial-to-mesenchymal transition. Oncogene 28: 422-430, 2009.

48. Chan CH, Lee SW, Li CF, Wang J, Yang WL, Wu CY, Wu J, Nakayama KI, Kang HY, Hung MC, et al: Deciphering the transcriptional complex critical for RhoA gene expression and cancer metastasis. Nat Cell Biol 12: 457-467, 2010.

This work is licensed under a Creative Commons Attribution-NonCommercial-NoDerivatives 4.0 International (CC BY-NC-ND 4.0) License. 